María Santamaría González, María Ángels Ruiz Mínguez, María Monsalud Arrebola Ramírez, Xavier Filella Pla, María José Torrejón Martínez, Daniel Morell García, Miguel Ángel Castaño López, Juan Antonio Allué Palacín, María Dolores Albaladejo Otón and Nuria Giménez Gómez*

\title{
An opportunity to emphasize the relevance of laboratory medicine
}

https://doi.org/10.1515/almed-2021-0029

Received September 29, 2020; accepted February 11, 2021; published online July 9, 2021

\section{Abstract}

Objectives: Clinical practice guidelines (CPGs) are recommendations based on a systematic review of scientific evidence that are intended to help healthcare professionals and patients make the best clinical decisions. CPGs must be evidence-based and are designed by multidisciplinary teams. The purpose of this study is to assess the topics related to the clinical laboratory addressed in CPGs and evaluate the involvement of laboratory professionals in the CPG development process.

Methods: A total of 16 CPGs recommended by the Spanish Society of Laboratory Medicine and/or retrieved from PubMed-Medline were included. A review of the information

\footnotetext{
*Corresponding author: Nuria Giménez Gómez, MD, PhD, Commission of Evidence-Based Laboratory Medicine, Spanish Society of Laboratory Medicine (SEQCML), Barcelona, Spain; Research Unit, Research Foundation, Mutua de Terrassa, University of Barcelona, Barcelona, Spain; Laboratory of Toxicology, Universitat Autònoma de Barcelona, Barcelona, Spain; and Commission of Evidence-Based Laboratory Medicine, International Federation of Clinical Chemistry (IFCC), Milan, Italy, E-mail: nuria.gimenez@uab.cat. https://orcid.org/ 0000-0002-0962-6216

María Santamaría González, Service of Clinical Biochemistry, Miguel Servet University Hospital, Zaragoza, Spain; and Commission of Evidence-Based Laboratory Medicine, Spanish Society of Laboratory Medicine (SEQC ${ }^{\mathrm{ML}}$ ), Barcelona, Spain

María Ángels Ruiz Mínguez, Commission of Evidence-Based Laboratory Medicine, Spanish Society of Laboratory Medicine $\left(\mathrm{SEQC}^{\mathrm{ML}}\right)$, Barcelona, Spain; and Service of Laboratory Medicine, Fundació Hospital de l'Esperit Sant, Santa Coloma de Gramenet, Barcelona, Spain

María Monsalud Arrebola Ramírez, Commission of Evidence-Based Laboratory Medicine, Spanish Society of Laboratory Medicine (SEOC ${ }^{\mathrm{ML}}$ ), Barcelona, Spain; and Laboratory Clinical Management Unit, Hospital de la Axarquía (AGSEMA), Málaga, Spain

Xavier Filella Pla, Commission of Evidence-Based Laboratory Medicine, Spanish Society of Laboratory Medicine (SEQC ${ }^{\mathrm{ML}}$ ),
}

provided in CPGs about 80 topics related to the clinical laboratory was performed. The authorship of laboratory professionals was assessed.

Results: On average, the 16 CPGs addressed 49\% (standard deviation [SD]: $11 \%$ ) of the topics evaluated in relation to the clinical laboratory. By order of frequency, CPGs contained information about $69 \%$ of postanalytical variables (SD: $20 \%$ ); $52 \%$ of preanalytical variables (SD: $11 \%$ ); and $43 \%$ of the analytical variables studied (SD: 18\%). Finally, half the CPGs included a laboratory professional among its authors.

Conclusions: CPGs frequently failed to provide relevant laboratory-related information. Laboratory professionals were co-authors in only half the CPGs. There is scope for improvement, and laboratory professionals should be included in multidisciplinary teams involved in the development of CPGs.

Keywords: biomarkers; clinical laboratory; clinical practice guidelines; evidence-based medicine; laboratory medicine.

Barcelona, Spain; Service of Biochemistry and Molecular Genetics (CDB), Hospital Clinic, IDIBAPS, Barcelona, Spain; and Commission of Evidence-Based Laboratory Medicine, International Federation of Clinical Chemistry (IFCC), Milan, Italy

María José Torrejón Martínez, Commission of Evidence-Based Laboratory Medicine, Spanish Society of Laboratory Medicine (SEQC ${ }^{\mathrm{ML}}$ ), Barcelona, Spain; and Clinical Biochemistry Management Unit (UGC), Hospital Clínico San Carlos, Madrid, Spain Daniel Morell García, Commission of Evidence-Based Laboratory Medicine, Spanish Society of Laboratory Medicine (SEQC ${ }^{\mathrm{ML}}$ ), Barcelona, Spain; and Service of Laboratory Medicine, Hospital Universitari Son Espases, Palma de Mallorca, Spain Miguel Ángel Castaño López, Commission of Evidence-Based Laboratory Medicine, Spanish Society of Laboratory Medicine $\left(\mathrm{SEQC}^{\mathrm{ML}}\right)$, Barcelona, Spain; and Service of Clinical Biochemistry, Hospital Clínico Universitario Juan Ramón Jiménez, Huelva, Spain Juan Antonio Allué Palacín, Commission of Evidence-Based Laboratory Medicine, Spanish Society of Laboratory Medicine (SEQC ${ }^{\mathrm{ML}}$ ), Barcelona, Spain; and Synlab Diagnosticos Globales, Sevilla, Spain

María Dolores Albaladejo Otón, Commission of Evidence-Based Laboratory Medicine, Spanish Society of Laboratory Medicine (SEQC ${ }^{\mathrm{ML}}$ ), Barcelona, Spain; and Service of Laboratory Testing and Clinical Biochemistry, Santa Lucía University Hospital, Cartagena, Spain 


\section{Introduction}

Clinical practice guidelines (CPGs) are defined as "recommendations informed by a systematic review of evidence and an assessment of the benefits and costs of alternative care options intended to optimize patient care" [1]. They are one of the most important tools for the optimization of clinical decisions [2].

CPGs for a specific condition must be developed by multidisciplinary teams composed of clinicians involved in the management of the disease. CPGs should be based on a scientific, rigorous, and transparent methodology and establish recommendations informed by the best evidence available [3]. In clinical practice, there is some confusion about the difference between CPGs and clinical protocols. Protocols are documents detailing the sequence of processes to be applied for the management of a specific health problem. They have a regulatory nature, are agreed and consider the resources available in the center where they are intended to be applied [4], whereas CPGs are at the top of the hierarchical pyramid of sources of evidence [5].

Since 2004, the Commission of Evidence-Based Laboratory Medicine of the Spanish Society of Laboratory Medicine $\left(\mathrm{SEQC}^{\mathrm{ML}}\right.$-) has coordinated a workgroup that disseminates the CPGs of the highest quality and/or its impact on clinical practice based on the opinions of the different SEQC ${ }^{\mathrm{ML}}$ - Commissions. This way, this commission contributes to the dissemination of quality scientific information.

It is worth mentioning that CPGs frequently fail to provide quality information about laboratory testing. A recent study assessing CPGs about prostate cancer screening with the prostate-specific antigen revealed that a laboratory professional had been involved in the development of only $9 \%$ of CPGs, and relevant information about laboratory tests was missing [6]. Surprisingly, a laboratory professional was not included in most multidisciplinary teams developing CPGs, even in those where laboratory testing plays an essential role in the management of a specific health problem.

The purpose of this study is to review the information related to the clinical laboratory contained in CPGs when recommendations for use of diagnostic and follow-up laboratory tests are included and evaluate the level of involvement of laboratory professionals in the CPGs' development process.

\section{Materials and methods}

\section{Source of information and search strategies}

A retrospective, descriptive, observational study was carried out to review CPGs published in the last five years (2015-2019). CPGs were retrieved from two sources of information: the website of the SEQC ${ }^{\mathrm{ML}}$, visited in December 2019, and the repository of health and medical literature PubMed.

The CPGs recommended by $\mathrm{SEQC}^{\mathrm{ML}}$ scientific commissions are available at http://www.seqc.es/es/gpc/.

The literature search was limited to papers related to laboratory medicine published in the last five years: (("Guideline" [PublicationType] OR "Guidelines as Topic”[Mesh] OR guideline ${ }^{\star}$ AND (“Clinical Laboratory Services”[Mesh] OR "Laboratories”[Mesh] OR laboratories)) AND ("Biomarkers”[Mesh] OR biomarkers) (search performed on November 29, 2019)

\section{Inclusion criteria}

CPGs were candidates for inclusion if they were guidelines, had been published in the previous five years, were written in English, and included recommendations for use of laboratory tests for diagnostic or therapeutic purposes. Redundant CPGs were only included once.

\section{Data collection}

CPGs were selected by two independent reviewers who applied the inclusion criteria described above. Discrepancies were solved by a third reviewer.

The CPGs selected were evaluated by peer review. The reviewers searched for relevant clinical laboratory data related to preanalytical, analytical, and postanalytical processes that may influence the clinical interpretation of test results. To such a purpose, we applied the checklist including 80 topics that should be considered for all laboratory tests included in a clinical practice guideline proposed by Aakre et al. [7] and adapted to the CPGs reviewed. Each study variable was converted into a dichotomous YES (1) or NO (0) variable, with being 0 the lowest score and 80 the highest.

The involvement of a laboratory medicine specialist in the guideline development process was assessed based on author affiliations. 'Laboratory medicine specialist' was defined as any professional with a specific university degree, with authority to exercise a specific function, and final personal accountability, who develops their professional practice in a laboratory, whether it was clinical or research practice.

\section{Statistical analysis}

Categorical variables are expressed as percentages. Normality of distribution was assessed by the Kolmogorov-Smirnov and Shapiro-Wilk tests. Continuous variables are expressed as a central tendency (mean or median) and dispersion standard deviation (SD) or interquartile 
range (IQR), based on their distribution. Qualitative variables were compared using $\chi^{2}$. Comparison of quantitative variables was performed by Student's t-test or the nonparametric Mann-Whitney U-test. All differences with a p-value of 0.05 were considered statistically significant. Statistical analyses were performed using IBM SPSS version 25 (Armonk, NY, USA).

\section{Results}

The CPGs selection process is displayed in Figure 1. A total of 165 CPGs were retrieved from PubMed, of which six met the inclusion criteria. The 143 papers that were not clinical practice guidelines but made reference to CPGs were excluded. A revision was performed of the $12 \mathrm{CPGs}$ recommended by the SEQC ${ }^{\mathrm{ML}}$ that met the inclusion criteria. Finally, 16 CPGs were selected for revision and evaluation (Table 1).

All phases of the analytical process were adequately represented in the CPGs reviewed which, on average, addressed 49\% (SD: 11\%) of the clinical laboratory topics evaluated in the study. By order of frequency, 69\% (SD: $20 \%$ ) of the postanalytical variables, $52 \%$ (SD: $11 \%$ ) of preanalytical variables, and 43\% (SD: 18\%) of the analytical variables studied were addressed in the CPGs.
The information provided in CPGs about each phase is detailed in Table 2 by the involvement of laboratory medicine specialists and by the source of information. A laboratory professional was involved in the development of $50 \%$ of the CPGs. An association was not observed between the laboratory-related information contained in a CPG and the involvement of a laboratory professional in the development process $(p=1.000)$. The source of information from which the CPG was selected was not associated with the laboratory topics addressed.

Table 3 summarizes the frequencies of appearance of the 80 topics investigated. In relation to the preanalytical phase, aspects related to sample requirements were most frequently missing. In the analytical phase, the least frequently addressed topic was the presence of analytical interferences.

\section{Discussion}

This study demonstrates that, despite the relevance of CPGs for the optimization of patient care, there is scope for improvement in the clinical laboratory. Thus, laboratory

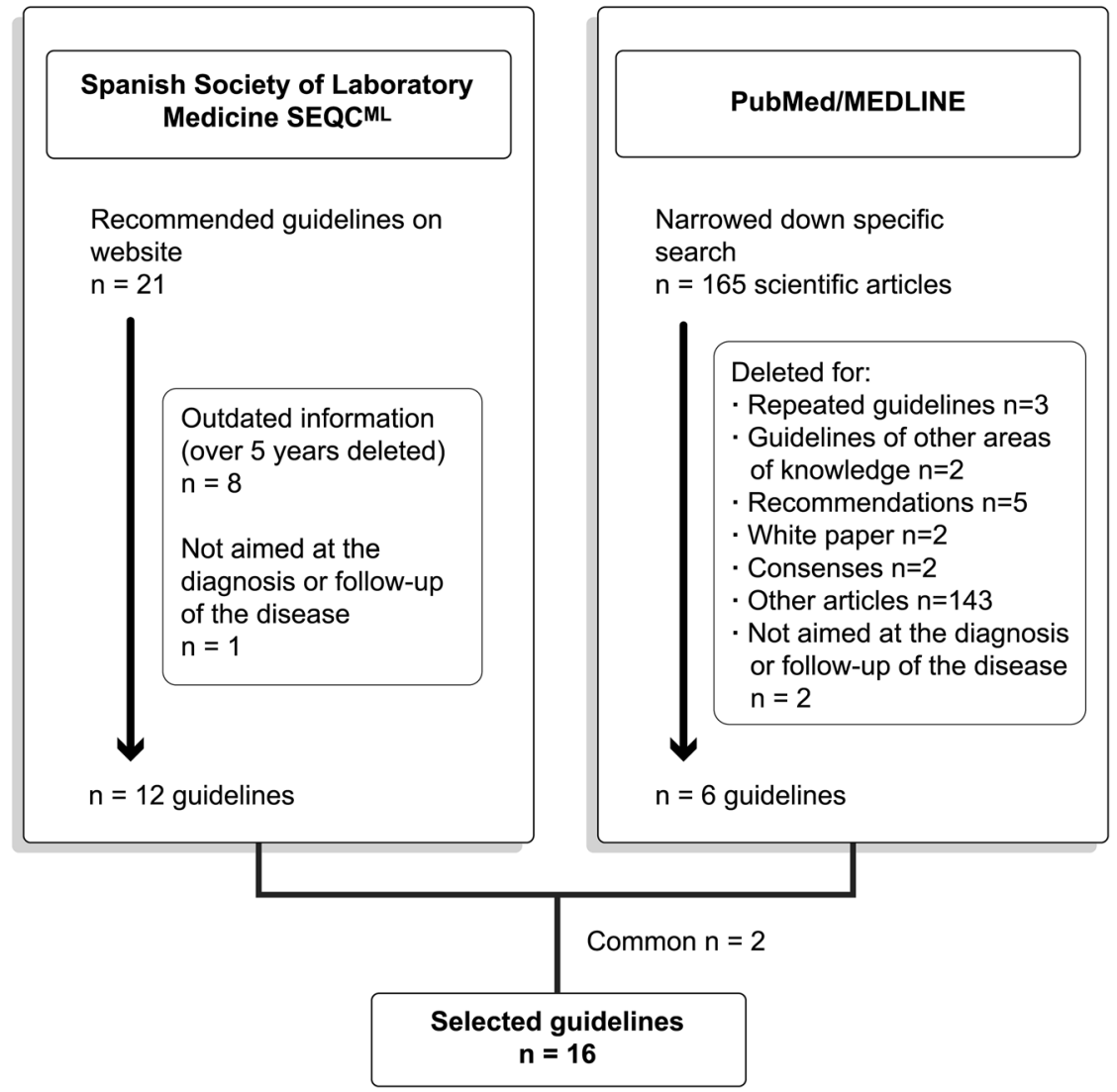

Figure 1: Clinical practice guideline selection process. 
Table 1: Clinical practice guidelines (CPGs) reviewed and percentage of CPGs that provide relevant data about the preanalytical, analytical and postanalytical phase.

\begin{tabular}{|c|c|c|c|c|c|}
\hline \multirow{2}{*}{$\begin{array}{l}\text { CPG reviewed }(n=16) \\
\text { (Maximum score possible) }\end{array}$} & \multicolumn{4}{|c|}{ Phases of the analytical process ${ }^{a}$} & \multirow{2}{*}{$\begin{array}{l}\text { Laboratory } \\
\text { professional } \\
n=16\end{array}$} \\
\hline & $\begin{array}{r}\text { Preanalytical } \\
\text { (33 points) }\end{array}$ & $\begin{array}{r}\text { Analytical } \\
(38 \\
\text { points })\end{array}$ & $\begin{array}{r}\text { Postanalytical } \\
\text { (9 points) }\end{array}$ & $\begin{array}{r}\text { Total } \\
(80 \text { points })\end{array}$ & \\
\hline $\begin{array}{l}\text { Clinical use of cancer biomarkers in epithelial ovarian } \\
\text { cancer: updated guidelines from the European Group on } \\
\text { Tumor Markers [8]. }\end{array}$ & $50 \%(16 / 32)$ & $\begin{array}{r}13 \% \\
(5 / 38)\end{array}$ & $78 \%(7 / 9)$ & $35 \%(28 / 79)$ & Yes \\
\hline $\begin{array}{l}\text { Clinical use of biomarkers in breast cancer: updated } \\
\text { guidelines from the European Group on Tumor Markers [9]. }\end{array}$ & $46 \%(12 / 26)$ & $\begin{array}{r}44 \% \\
(11 / 25)\end{array}$ & $50 \%(3 / 6)$ & $46 \%(26 / 57)$ & Yes \\
\hline $\begin{array}{l}\text { Revised American Thyroid Association Guidelines for the } \\
\text { management of medullary thyroid carcinoma [10]. }\end{array}$ & $61 \%(17 / 28)$ & $\begin{array}{r}68 \% \\
(15 / 22)\end{array}$ & $78 \%(7 / 9)$ & $66 \%(39 / 59)$ & Yes \\
\hline $\begin{array}{l}\text { Congenital adrenal hyperplasia due to steroid } \\
\text { 21-hydroxylase deficiency: An Endocrine Society Guideline } \\
\text { [11]. }\end{array}$ & $66 \%(21 / 32)$ & $\begin{array}{r}68 \% \\
(15 / 22)\end{array}$ & $67 \%(6 / 9)$ & $67 \%(42 / 63)$ & No \\
\hline $\begin{array}{l}\text { Clinical practice guidelines for the care of girls and women } \\
\text { with turner syndrome [12]. }\end{array}$ & $42 \%(11 / 26)$ & $\begin{array}{r}73 \% \\
(8 / 11)\end{array}$ & $60 \%(3 / 5)$ & $52 \%(22 / 42)$ & Yes \\
\hline $\begin{array}{l}\text { European Guidelines on cardiovascular disease prevention } \\
\text { in clinical practice [13]. }\end{array}$ & $48 \%(12 / 25)$ & $\begin{array}{r}52 \% \\
(15 / 29)\end{array}$ & $56 \%(5 / 9)$ & $51 \%(32 / 63)$ & No \\
\hline Guideline on the management of blood cholesterol [14]. & $48 \%(16 / 33)$ & $\begin{array}{r}42 \% \\
(16 / 38)\end{array}$ & $89 \%(8 / 9)$ & $50 \%(40 / 80)$ & Yes \\
\hline Guidelines for the management dyslipidaemias [15]. & $58 \%(19 / 33)$ & $\begin{array}{r}45 \% \\
(17 / 38)\end{array}$ & $89 \%(8 / 9)$ & $55 \%(44 / 80)$ & Yes \\
\hline $\begin{array}{l}\text { Guidelines for the diagnosis and treatment of acute and } \\
\text { chronic heart failure [16]. }\end{array}$ & $27 \%(9 / 33)$ & $\begin{array}{r}18 \% \\
(7 / 38)\end{array}$ & $56 \%(5 / 9)$ & $26 \%(21 / 80)$ & No \\
\hline $\begin{array}{l}\text { Guidelines for the management of acute coronary syn- } \\
\text { dromes in patients presenting without persistent } \\
\text { ST-segment elevations [17]. }\end{array}$ & $76 \%(22 / 29)$ & $\begin{array}{r}33 \% \\
(11 / 33)\end{array}$ & $89 \%(8 / 9)$ & $58 \%(41 / 71)$ & No \\
\hline Chronic kidney disease in adults [18]. & $42 \%(14 / 33)$ & $\begin{array}{r}39 \% \\
(15 / 38)\end{array}$ & $89 \%(8 / 9)$ & $46 \%(37 / 80)$ & No \\
\hline Anemia management in chronic kidney disease [19]. & $69 \%(20 / 29)$ & $\begin{array}{r}42 \% \\
(13 / 31)\end{array}$ & $89 \%(8 / 9)$ & $59 \%(41 / 69)$ & No \\
\hline $\begin{array}{l}\text { Updated molecular testing guideline for the selection of } \\
\text { lung cancer patients for treatment with targeted tyrosine } \\
\text { kinase inhibitors [20]. }\end{array}$ & $55 \%(18 / 33)$ & $\begin{array}{r}13 \% \\
(5 / 38)\end{array}$ & $44 \%(4 / 9)$ & $34 \%(27 / 80)$ & Yes \\
\hline $\begin{array}{l}\text { Biomarkers in nonsmall cell lung cancers: Indian } \\
\text { consensus guidelines for molecular testing [21]. }\end{array}$ & $48 \%(14 / 29)$ & $\begin{array}{r}53 \% \\
(17 / 32)\end{array}$ & $89 \%(8 / 9)$ & $56 \%(39 / 70)$ & Yes \\
\hline $\begin{array}{l}\text { ESMO consensus guidelines for the management of pa- } \\
\text { tients with metastatic colorectal cancer [22]. }\end{array}$ & $45 \%(10 / 22)$ & $\begin{array}{r}37 \% \\
(10 / 27)\end{array}$ & $22 \%(2 / 9)$ & $38 \%(22 / 58)$ & No \\
\hline $\begin{array}{l}\text { Human epidermal growth factor receptor } 2 \text { testing in breast } \\
\text { cancer [23]. }\end{array}$ & $48 \%(11 / 23)$ & $\begin{array}{r}48 \% \\
(10 / 21)\end{array}$ & $56 \%(5 / 9)$ & $49 \%(26 / 53)$ & No \\
\hline Total & $52 \%$ & $43 \%$ & $69 \%$ & $49 \%$ & $50 \%(8 / 16)$ \\
\hline
\end{tabular}

${ }^{\text {a}}$ Results are expressed as \% (absolute number of addressed topics related to the laboratory/absolute number of relevant laboratory topics).

medicine specialists should be invited to join multidisciplinary guideline development teams. In addition, more detailed information should be provided in CPGs about specific aspects of diagnostic laboratory tests, which are essential in clinical decision-making [24-26].

The CPGs including recommendations for use of laboratory tests for diagnostic or follow-up purposes only provided half the relevant information related to laboratory testing. Moreover, specific aspects such as preanalytical sample requirements or analytical interferences were hardly mentioned. On the other hand, all analytical phases i.e. preanalytical, analytical, and postanalytical, were considered. It is important to be aware of the limitations and potential errors that may occur across the different laboratory testing processes, as they may have a negative impact on the patient [27]. Laboratory testing is an essential part of clinical decision-making, and most CPGs include recommendations for use of laboratory tests. However, 
Table 2: Topics addressed in clinical practice guidelines related to the different analytical phases by authorship and source of information.

Information provided in clinical practice guidelines

(\% = number of laboratory topics addressed/maximum number of topics)

CPGs developed with the involvement of laboratory medicine specialists.

\begin{tabular}{lrrr}
\hline $\mathbf{n = 1 6}$ & Yes (n=8) & No (n=8) & $\begin{array}{r}\mathbf{p}- \\
\text { Value }\end{array}$ \\
\hline Preanalytical phase. Mean (SD) $^{\mathrm{a}}$ & $51(6)$ & $53(16)$ & 0.799 \\
Analytical phase. Mean (SD) $^{\mathrm{a}}$ & $44(22)$ & $42(15)$ & 0.859 \\
Postanalytical phase. Median & $78(37)$ & $62(33)$ & 0.705 \\
$(\mathrm{IQR})^{\mathrm{b}}$ & & & \\
Total analytical process. Mean $_{(S D)^{\mathrm{a}}}$ & $49(11)$ & $49(13)$ & 1.000 \\
\hline
\end{tabular}

\begin{tabular}{|c|c|c|c|}
\hline \multicolumn{4}{|l|}{ Source of information } \\
\hline$n=14^{d}$ & $\begin{array}{r}\text { SEQC-ML } \\
(n=10)\end{array}$ & $\begin{array}{r}\text { PubMed } \\
(n=4)\end{array}$ & $\begin{array}{r}\mathrm{p}- \\
\text { Value }\end{array}$ \\
\hline Preanalytical phase. Mean (SD) ${ }^{\mathrm{a}}$ & 54 (15) & $49(4)$ & 0.382 \\
\hline Analytical phase. Mean (SD) ${ }^{\mathrm{a}}$ & $48(17)$ & $38(18)$ & 0.342 \\
\hline $\begin{array}{l}\text { Postanalytical phase. Median } \\
(I Q R)^{b}\end{array}$ & $84(30)$ & $50(53)$ & 0.089 \\
\hline $\begin{array}{l}\text { Total analytical process. Mean } \\
(\text { SD) }\end{array}$ & $53(12)$ & $44(10)$ & 0.214 \\
\hline $\begin{array}{l}\text { Authorship of laboratory medi- } \\
\text { cine specialists }^{c}\end{array}$ & $40 \%$ & $67 \%$ & 0.298 \\
\hline
\end{tabular}

SD, standard deviation; IQR, interquartile range. ${ }^{\text {a }}$ Student's t-test.

${ }^{b}$ Nonparametric Mann-Whitney U-test. ${ }^{C} X^{2}$-test. ${ }^{d}$ Repeated CPGs were considered only once for statistical analysis.

information about some relevant aspects of laboratory processes is missing $[6,7]$ despite being necessary for an appropriate interpretation of results [27]. The quality of CPGs could be improved by addressing relevant aspects of laboratory processes $[6,7]$.

A laboratory professional had been involved in the development of half the CPGs studied, which is a low rate, considering that we selected CPGs that recommended specific laboratory tests for diagnosis and follow-up of disease. Surprisingly, the rate of participation of laboratory professionals in the CPG development process was even lower in the few studies where this topic was addressed $[6,7]$. All the professionals involved in the management of a specific disease should take part in the development of CPGs for that disease, which would guarantee multidisciplinarity. It is likely that relevant aspects about laboratory testing are not appropriately addressed when a laboratory professional hasn't taken part in the recommendations for use of a laboratory test in a guideline [28]. Additionally, the quality of CPGs would improve if evidence about the clinical relevance
Table 3: Checklist employed to review clinical practice guidelines (CPGs) (modified by Aakre et al. [7]).

\begin{tabular}{|c|c|c|}
\hline Preanalytical phase & & $\begin{array}{r}\text { CPG } \\
\text { inclusion }\end{array}$ \\
\hline \multirow{4}{*}{$\begin{array}{l}\text { Description of the } \\
\text { target population }\end{array}$} & Age & $88 \%$ \\
\hline & Sex & $84 \%$ \\
\hline & Disease & $96 \%$ \\
\hline & Specific diseases & $94 \%$ \\
\hline \multirow{6}{*}{$\begin{array}{l}\text { Indications for } \\
\text { biomarker use }\end{array}$} & Monitoring & $84 \%$ \\
\hline & Frequency of testing & $68 \%$ \\
\hline & Diagnosis & $83 \%$ \\
\hline & Prognosis & $88 \%$ \\
\hline & Screening & $57 \%$ \\
\hline & Auto-monitoring & $50 \%$ \\
\hline \multirow[t]{8}{*}{ Clinical performance } & Sensitivity & $65 \%$ \\
\hline & Specificity & $59 \%$ \\
\hline & ROC curve & $6 \%$ \\
\hline & Added value of the biomarker & $81 \%$ \\
\hline & $\begin{array}{l}\text { Comparison with other related } \\
\text { biomarkers }\end{array}$ & $69 \%$ \\
\hline & $\begin{array}{l}\text { Probability of diagnosis after the } \\
\text { test }\end{array}$ & $52 \%$ \\
\hline & Positive outcome of testing & $31 \%$ \\
\hline & Negative outcome of testing & $28 \%$ \\
\hline \multirow[t]{5}{*}{$\begin{array}{l}\text { Multiple approach } \\
\text { with other biomarkers }\end{array}$} & $\begin{array}{l}\text { Inclusion in a panel with other } \\
\text { biomarkers }\end{array}$ & $71 \%$ \\
\hline & Sensitivity (panel) & $39 \%$ \\
\hline & Specificity (panel) & $39 \%$ \\
\hline & ROC curve (panel) & $0 \%$ \\
\hline & Added value of the panel & $57 \%$ \\
\hline \multirow[t]{10}{*}{ Sample requirements } & Fasting & $35 \%$ \\
\hline & Time from the clinical event & $28 \%$ \\
\hline & Patient position & $5 \%$ \\
\hline & Circadian rhythm & $10 \%$ \\
\hline & Type of sample & $75 \%$ \\
\hline & Sample transportation & $9 \%$ \\
\hline & Centrifugation & $0 \%$ \\
\hline & $\begin{array}{l}\text { Sample pretreatment (maximum } \\
\text { delay) }\end{array}$ & $19 \%$ \\
\hline & $\begin{array}{l}\text { Maximum time of storage at the } \\
\text { specified temperature }\end{array}$ & $6 \%$ \\
\hline & $\begin{array}{l}\text { Maximum no. of freezing- } \\
\text { thawing cycles }\end{array}$ & $3 \%$ \\
\hline
\end{tabular}

\begin{tabular}{llr}
\hline Analytical phase & & $\begin{array}{r}\text { CPG } \\
\text { inclusion }\end{array}$ \\
\hline Method & Recommended method & $72 \%$ \\
& Standardization & $41 \%$ \\
& Traceability to the method of & $38 \%$ \\
& reference & \\
& Heterogeneity of biomarkers & $50 \%$ \\
Analytical & Limit of detection & $17 \%$ \\
interferences & Lipemia & $6 \%$ \\
& Hemolysis & $0 \%$ \\
& Bilirubin & $0 \%$ \\
& Monoclonal paraproteins & $6 \%$ \\
& Heterophile antibodies & $16 \%$ \\
& Endogenous antibodies & $15 \%$
\end{tabular}


Table 3: (continued)

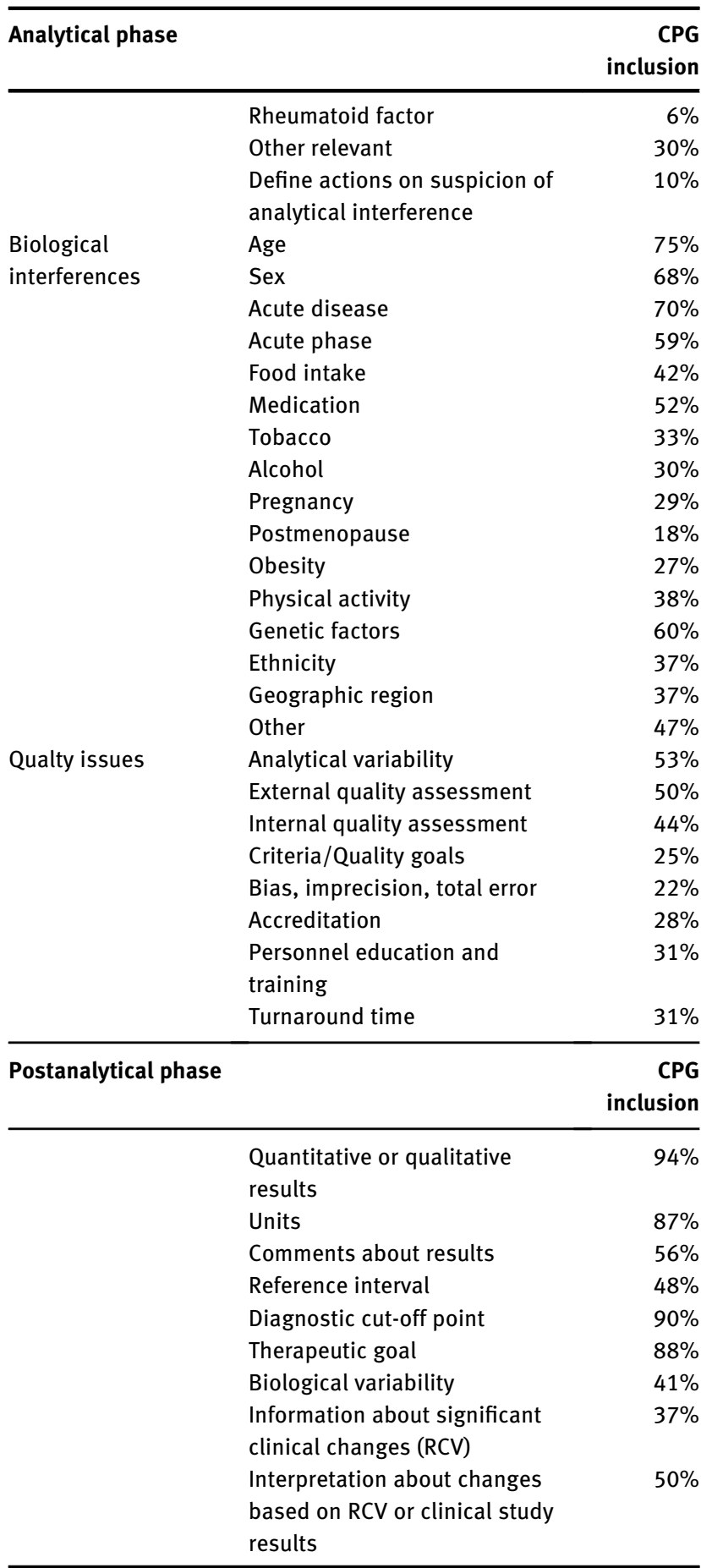

Contains 80 relevant topics related to the clinical laboratory distributed across the preanalytical, analytical and postanalytical phase. If a topic was not considered relevant to the evaluation of the CPGs, the nominator was adjusted for percentage calculation.

of laboratory tests was provided, which would also contribute to optimize the use of the clinical laboratory and the usefulness of new biomarkers [24]. The complexity of laboratory testing demands a good understanding of CPGs and the knowledge of the limitations of the analytical methods by laboratory staff, who also help interpreting and using laboratory data [28]. The activity of laboratory professionals is interconnected with all medical disciplines and provides crucial support in healthcare [26]. Therefore, it is essential to raise awareness about the relevance of clinical advice and the involvement of laboratory professionals in patient care. Moreover, laboratory medicine specialists should more frequently join the multidisciplinary teams that develop, review, disseminate, and use CPGs.

Paradoxically, unlike previous studies, in our study, the involvement of laboratory medicine specialists in the guideline development process did not lead to the increased attention given to laboratory-related topics [7, 28]. Thus, Akree et al. documented that more information is provided about the type of sample, shipping, and other analytical aspects in the CPGs developed by a multidisciplinary team, including a laboratory medicine specialist [7]. The British Thoracic Society guidelines for the investigation of unilateral pleural effusion in adults also recommend a resource to laboratory medicine specialists for improving the use and interpretation of laboratory tests by clinicians [28]. No statistically significant differences were observed in laboratory-related information quality and the involvement of a laboratory medicine specialist in the CPG development process. This may be due to our selection method. The sample was composed of high-quality CPGs recommended by the SEQC ${ }^{\mathrm{ML}}$ Commission for their relevance and usefulness for the clinical laboratory or retrieved from a literature search on PubMed limited to clinical laboratory topics. We postulate that although the authorship of laboratory professionals is not recognized in a CPG, they may have been involved in the development process as external collaborators, advisers, expert consultants, and/or reviewers, as many aspects related to laboratory testing are addressed in these CPGs.

Laboratory medicine professionals are specialists in the biochemical and biological interactions that characterize human diseases. Their professional experience with laboratory testing and its clinical relevance position empowered them as reliable consultants with an in-depth knowledge of preanalytical requirements and of the clinical relevance and interpretation of test results. Hence, laboratory professionals provide supplementary information to the one recorded in medical reports and guarantee quality patient care [29]. Additionally, they contribute to reduce potential diagnostic errors and improve the quality of healthcare and health services [30]. Laboratory medicine can be occasionally perceived as a neglected discipline, which is clearly seen in the high proportion of papers showing a lack of understanding 
about the relevance of laboratory testing [25]. Laboratory medicine specialists should increase their visibility and get more actively involved in clinical teams. This would raise awareness about the relevance of their role and the added value they can bring as consultants in patientcentered care.

$\mathrm{SEQC}^{\mathrm{ML}}$, as a scientific society, prioritizes access to high-quality information and recommends CPGs grouped by fields of medicine. These CPGs are regularly updated to incorporate recent evidence and are intended to meet the needs of laboratory medicine specialists. Health professionals find it difficult to cope with the flood of biomedical information published every month since searching for high-quality publications is time-consuming [31]. The availability of a website providing access to relevant CPGs facilitates the selection of quality publications by laboratory professionals [32]. Thus, scientific societies meet one of their main goals: managing and promoting the generation, uptake, dissemination, and handling of knowledge [33]. The publication of CPGs recommended by the $\mathrm{SEQC}^{\mathrm{ML}}$ on its website is complemented by other multiple initiatives launched in collaboration with other scientific societies and raises the visibility of the role of laboratory medicine specialists.

Thus, with the publication of quality CPGs on its website, the $\mathrm{SEQC}^{\mathrm{ML}}$ facilitates the translation of CPGs recommendations into clinical practice, including laboratory medicine practice [34], hence giving easy, rapid access to CPGs previously reviewed and organized by topics. The need to promote the implementation of CPGs by laboratory medicine specialists is recognized by the scientific community, and the benefits of their involvement in CPG development and implementation are widely known [35]. However, laboratory medicine specialists have difficulties to access and join multidisciplinary CPG development teams.

The reduced sample of CPGs reviewed may be a limitation of this study. We retrieved all CPGs recommended by the $\mathrm{SEQC}^{\mathrm{ML}}$ and a literature search of CPGs addressing topics related to the clinical laboratory was performed. Therefore, despite the sample bias inherent to CPG selection, the sample was adequate for the purposes of this study.

\section{Conclusions}

The major contribution of this study is that it emphasizes the relevant role that laboratory medicine specialists and scientific societies play in the dissemination of scientific information by fostering collaboration between panels of experts that recommend CPGs which include relevant information to each particular field of knowledge. We recommend laboratory medicine specialists to reinforce their role as consultants, foster interdepartmental cooperation, and draw attention to how a clinical laboratory works, to avoid errors and improve patient care [36]. Laboratory professionals are responsible for ensuring that all phases of laboratory testing are performed appropriately, from the preanalytical and postanalytical phase where most errors concentrate, and which specialists and patients are less aware, through the analytical phase, where errors are less frequent (13-32\%) [37].

Moreover, this study draws attention to points of improvement in the development of CPGs. The development of a CPG is an example of an interdisciplinary project where laboratory specialists can cooperate and share their expertise. The involvement of laboratory medicine specialists in interdisciplinary projects raises awareness about their relevant roles and functions. Further studies are needed to investigate the level of implementation of CPGs in daily laboratory practice, assess their impact on healthcare quality, and evaluate laboratory professionals' involvement in the development and application of CPGs.

Research funding: None declared.

Author contributions: All authors accept responsibility for the contents of the manuscript submitted for publication. MSG, MARM, MMAR, XF, MJTM, and NG involved in the conception and design of the study. MSG, MARM, MMAR, and NG selected and reviewed clinical practice guidelines. MSG and NG drafted the first version of the manuscript and performed the statistical analysis. All authors participated in the updating of the clinical practice guidelines recommended by the Spanish Society of Laboratory Medicine published on its website, as well as in the interpretation of results and revision of the final manuscript. MSG and NG are the guarantors of the study. All authors have accepted responsibility for the entire content of this manuscript and approved its submission.

Competing interests: The authors declare no conflict of interest.

Ethical approval: Not applicable.

\section{References}

1. Institute of Medicine (U.S.A). Committee on standards for developing trustworthy clinical practice guidelines. In: Graham R, Mancher M, Wolman DM, Greenfield S, Steinberg E, editors. Clinical practice guidelines we can trust. Washington, D.C.: The National Academies Press; 2011. Available from: http://www.nap. edu/read/13058/chapter/1 [Accessed 6 Jul 2020]. 
2. Djulbegovic B, Guyatt G. Evidence vs consensus in clinical practice guidelines. J Am Med Assoc 2019;322:725-6.

3. Rabi DM, Kunneman M, Montori VM. When guidelines recommend shared decision-making. J Am Med Assoc 2020;323:1345-6.

4. Gracia García S, Giménez Gómez N, Ruiz Mínguez A. Medicina basada en la evidencia - guías de práctica clínica. Bioquímica Patol Clínica 2006;69:34-7.

5. Florkowski C, Don-Wauchope A, Gimenez N, Rodriguez-Capote K, Wils J, Zemlin A. Point-of-care testing (POCT) and evidence-based laboratory medicine (EBLM) - does it leverage any advantage in clinical decision making? Crit Rev Clin Lab Sci 2017;54:471-94.

6. Filella X, Albaladejo MD, Allué JA, Castaño MA, Morell-Garcia D, Ruiz MA, et al. Prostate cancer screening: guidelines review and laboratory issues. Clin Chem Lab Med 2019;57:1474-87.

7. Aakre KM, Langlois MR, Watine J, Barth JH, Baum H, Collinson P, et al. Critical review of laboratory investigations in clinical practice guidelines: proposals for the description of investigation. Clin Chem Lab Med 2013;51:1217-26.

8. Sölétormos G, Duffy MJ, Othman Abu Hassan S, Verheijen RH, Tholander B, Bast RC, et al. Clinical use of cancer biomarkers in epithelial ovarian cancer: updated guidelines from the European Group on Tumor Markers. Int J Gynecol Canc 2016;26:43-51.

9. Duffy MJ, Harbeck N, Nap M, Molina R, Nicolini A, Senkus E, et al. Clinical use of biomarkers in breast cancer: updated guidelines from the European Group on Tumor Markers (EGTM). Eur J Canc 2017;75:284-98.

10. Wells SA Jr, Asa SL, Dralle H, Elisei R, Evans DB, Gagel RF, et al. Revised American Thyroid Association guidelines for the management of medullary thyroid carcinoma. Thyroid 2015;25: 567-610.

11. Speiser PW, Arlt W, Auchus RJ, Baskin LS, Conway GS, Merke DP, et al. Congenital adrenal hyperplasia due to steroid 21-hydroxylase deficiency: an Endocrine Society Clinical Practice Guideline [published correction appears in J Clin Endocrinol Metab. 2019;104: 39-40]. J Clin Endocrinol Metab 2018;103:4043-88.

12. Gravholt $\mathrm{CH}$, Andersen NH, Conway GS, Dekkers OM, Geffner ME, Klein KO, et al. Clinical practice guidelines for the care of girls and women with Turner syndrome: proceedings from the 2016 Cincinnati International Turner Syndrome Meeting. Eur J Endocrinol 2017;177:G1-70.

13. Piepoli MF, Hoes AW, Agewall S, Albus C, Brotons C, Catapano AL, et al. 2016 European guidelines on cardiovascular disease prevention in clinical practice: the sixth joint task force of the European society of cardiology and other societies on cardiovascular disease prevention in clinical practice (constituted by representatives of 10 societies and by invited experts) developed with the special contribution of the European association for cardiovascular prevention \& rehabilitation (EACPR). Atherosclerosis 2016;252:207-74.

14. Grundy SM, Stone NJ, Bailey AL, Beam C, Birtcher KK, Blumenthal RS, et al. 2018 AHA/ACC/AACVPR/AAPA/ABC/ACPM/ADA/AGS/ APhA/ASPC/NLA/PCNA guideline on the management of blood cholesterol: a report of the American college of cardiology/ American heart association task force on clinical practice guidelines. Circulation 2019;139:e1082-143.

15. Catapano AL, Graham I, De Backer G, Wiklund O, Chapman MJ, Drexel H. 2016 ESC/EAS guidelines for the management of dyslipidaemias. Kardiol Pol 2016;74:1234-318.

16. Ponikowski P, Voors AA, Anker SD, Bueno H, Cleland JGF, Coats AJS, et al. 2016 ESC guidelines for the diagnosis and treatment of acute and chronic heart failure: the task force for the diagnosis and treatment of acute and chronic heart failure of the European Society of Cardiology (ESC). Developed with the special contribution of the Heart Failure Association (HFA) of the ESC. Eur Heart J 2016;37:2129-200.

17. Roffi M, Patrono C, Collet JP, Mueller C, Valgimigli M, Andreotti F, et al. 2015 ESC guidelines for the management of acute coronary syndromes in patients presenting without persistent ST-segment elevation: task force for the management of acute coronary syndromes in patients presenting without persistent ST-segment elevation of the European Society of Cardiology (ESC). Eur Heart J 2016;37:267-315.

18. National Institute for Health and Care Excellence (NICE). Chronic kidney disease in adults: assessment and management. London: National Institute for Health and Care Excellence (UK); 2015.

19. National Clinical Guideline Centre (UK). Anaemia management in chronic kidney disease: partial update 2015. London: Royal College of Physicians (UK); 2015.

20. Lindeman NI, Cagle PT, Aisner DL, Arcila ME, Beasley MB, Bernicker EH, et al. Updated molecular testing guideline for the selection of lung cancer patients for treatment with targeted tyrosine kinase inhibitors: guideline from the College of American Pathologists, the International Association for the Study of Lung Cancer, and the Association for Molecular Pathology. J Mol Diagn 2018;20:129-59.

21. Prabhash K, Advani SH, Batra U, Biswas B, Chougule A, Ghosh M, et al. Biomarkers in non-small cell lung cancers: Indian consensus guidelines for molecular testing. Adv Ther 2019;36:766-85.

22. Van Cutsem E, Cervantes A, Adam R, Sobrero A, Van Krieken JH, Aderka D, et al. ESMO consensus guidelines for the management of patients with metastatic colorectal cancer. Ann Oncol 2016;27: 1386-422.

23. Wolff AC, Hammond MEH, Allison KH, Harvey BE, Mangu PB, Bartlett JMS, et al. Human epidermal growth factor receptor 2 testing in breast cancer: American Society of Clinical Oncology/ College of American Pathologists. Clinical practice guideline focused update. Arch Pathol Lab Med 2018;142:1364-82.

24. Horvath AR. From evidence to best practice in laboratory medicine. Clin Biochem Rev 2013;34:47-60.

25. Lippi G. The irreplaceable value of laboratory diagnostics: four recent tests that have revolutionized clinical practice. EJIFCC 2019;30:7-13.

26. Plebani M, Laposata M, Lippi G. Driving the route of laboratory medicine: a manifesto for the future. Intern Emerg Med 2019;14: 337-40.

27. Plebani M. Exploring the iceberg of errors in laboratory medicine. Clin Chim Acta 2009;404:16-23.

28. Lapworth R, Tarn AC. Commentary on the British Thoracic Society guidelines for the investigation of unilateral pleural effusion in adults. Ann Clin Biochem 2006;43:17-22.

29. Sociedad Española de Bioquímica Clínica y Patología Molecular, editor. Funciones del facultativo del laboratorio clínico. El laboratorio Clínico del siglo XXI. Barcelona; 2006.

30. Taylor JR, Thompson PJ, Genzen JR, Hickner J, Marques MB. Opportunities to enhance laboratory professionals' role on the diagnostic team. Lab Med 2017;48:97-103.

31. Smith R. A POEM a week for the BMJ. BMJ 2002;325:983.

32. García Moreira V, Giménez Gómez N, Torrejón Martínez MJ, Allué Palacín JA, Filella Pla X, Ruiz Mínguez MA. Recursos y fuentes de información útiles en la práctica de la medicina de laboratorio basado en la evidencia. Rev Lab Clin 2017;10:21-30. 
33. Prados Castillejo JA. Las sociedades científicas y la gestión del conocimiento, un paso más allá del desarrollo profesional continuo. Atención Primaria 2010;42:338-41.

34. Conklin J, Farrell B, Suleman S. Implementing deprescribing guidelines into frontline practice: barriers and facilitators. Res Soc Adm Pharm 2019;15:796-800.

35. Kahn SE, Jones PM, Chin AC, Christenson RH. Defining the path forward: guidance for laboratory medicine guidelines. EJIFCC 2015;26:158-67.
36. Plebani M, Bonini P. Wrong biochemistry results. Interdepartmental cooperation may help avoid errors in medical laboratories. BMJ 2002;324:423-4.

37. Bonini P, Plebani M, Ceriotti F, Rubboli F. Errors in laboratory medicine. Clin Chem 2002;48:691-8.

Article Note: The original article can be found here: https://doi.org/ 10.1515/almed-2020-0094. 